\title{
Parameters Used For Objective Acoustic Vocal Analysis In Paediatric Patients with Bronchiectasis
}

\author{
Omer Faruk Calim¹, Yavuz Selim Yıldırım², Özge Gedik², Erkan Çakır, Orhan Özturan \\ 1: Bezmialem Vakif University, Medical faculty, Department of Otolaryngology Head and Neck Surgery, Istanbul, Turkey - ORCID ID: 0000-0002-0010-9028 \\ 2: Dogus University, Medical Faculty, ENT Department, Istanbul, Turkey - ORCID ID: 0000-0001-9796-0822 \\ 3. Bezmialem Vakif University, Health Science Faculty, Department of Audiology, Istanbul, Turkey - ORCID ID: 0000-0003-1315-9080 \\ 4. Bezmialem Vakif University, Medical Faculty, Department of Pediatrics, Istanbul, Turkey - ORCID ID: 0000-0002-6129-8627 \\ 5. Bezmialem Vakif University, Medical faculty, Department of Otolaryngology Head and Neck Surgery, Istanbul, Turkey - ORCID ID: 0000-0003-2680-7323
}

\begin{abstract}
Parameters Used For Objective Acoustic Vocal Analysis In Paediatric Patients with Bronchiectasis

Objectives: Bronchiectasis typically manifests with persistent coughing, pus-filled discharge, lethargy, dyspnoea, sternal pain, haemoptysis and dilated airways. Such symptoms can adversely affect the quality of life and voice characteristics of sufferers from bronchiectasis. This study had as its objective the objective comparison of vocal differences between healthy children and those with bronchiectasis.
\end{abstract}

Methods: The parameters examined were jitter, percentage jitter, shimmer, noise-to-harmonics ratio (NHR), voice turbulence index (VTI), and soft phonation index
(SPI). Data from the cases and controls were compared by means of paired t-tests.

Results: The mean age for the cases was 11.53 years. In terms of absolute jitter, percentage jitter, shimmer and SPI, no differences were detected at the level of statistical significance. However, the NHR and VTI did differ between cases and controls at the level of statistical significance.

Conclusion: The research reveals that an holistic approach has an influence on patient outcomes. Sufferers from bronchiectasis experience vocal alterations, especially with respect to NHR and VTI, the values of which were raised in comparison with healthy controls.

Key words: Acoustic analysis, voice, hoarseness, bronchiectasis

\section{Introduction}

The usual classification of bronchiactasis is into those cases secondary to cystic fibrosis (CF) and those not caused by $\mathrm{CF}$ (=NCFB) [1]. Bronchiectasis is characterised as a condition in which persistent long term inflammatory responses provoked by non-clearance of particular pathogens or recurrent/persistent infection in the bronchi lead to irreversible dilatation of the airways [2]. The aetiology of bronchiectasis ranges from repeated respiratory infective episodes to the less common immunodeficiency syndromes. A key descriptor is "irreversible dilatation of pe-

ripheral airways", and the diagnosis is typically confirmed radiologically through high-resolution computed tomography (HRCT) imaging of the thorax [3].

Bronchiectasis is known to produce persistent coughing, pus-filled discharge, lethargy, dyspnoea, sternal pain, haemoptysis and dilated airways. Pulmonary function can also deteriorate over time. These factors lead to a poorer quality of life for patients. However, managing bronchiectasis judiciously may increase the quality of life (QoL), alongside making exacerbations less severe and less common, and helping prevent permanent reductions in lung function [4-5].

Correspondence: Assistant Prof. Omer Faruk Calim, MD

Bezmialem Vakif University, Faculty of Medicine, Department of Otorhinolaryngology and Head and Neck Surgery Istanbul, Adnan Menderes Bulvarı Vatan Caddesi 34093 Fatih/Istanbul. Phone: +90 212453170080 TURKEY

e-mail: omercalim@yahoo.com

Received: January 20, 2019; Accepted: February 16, 2019
Online available at: www.entupdates.org

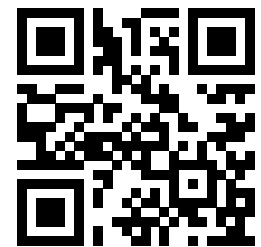


Whilst it is known that NCFB has effects on the voice through abnormal functioning of the glottis, exactly how this occurs is not yet understood, nor is it known which type of vocal analysis is most useful.

This study aimed to compare, on an objective basis, vocal quality in children, both healthy and with bronchiectasis.

\section{Materials and Methods}

Ethical approval was obtained from the Institutional Review Board of the Medical Centre. Parents provided informed consent for all child participants.

37 paediatric cases of bronchiectasis were enrolled with a mean age of 11.53 years (range 7-13), from an initial sample of 125 patients under follow-up by the paediatric pulmonology department. Healthy controls were also recruited, with a mean age of 9.88 years (range $7-13$ ). The research followed a prospective, controlled study design and took place at tertiary referral centre.

\section{Inclusion and exclusion criteria}

The cases were all children aged 7-13 years with known bronchiectasis and no co-morbidity in terms of cerebral injury, auditory function, autistic spectrum disorder or learning disability. In each case there were clinical and radiological findings consistent with bronchiectasis and sweat testing was negative. Cases where sweat test indicated CF, vocal cord lesions existed or who were outside the age range of 7-13 were excluded from both control and cases groups.

The parameters examined were jitter, percentage jitter, shimmer, noise-to-harmonics ratio (NHR), voice turbulence index (VTI), and soft phonation index (SPI).

\section{Objective measurements used for vocal analysis}

T-tests were used to compare data from the case (bronchiectasis) and control (healthy) groups. The analysis and recording of representative speech was accomplished with Multi-Dimensional Voice Program (MDVP) software (Model 4500, KayPENTAX, Lincoln Park, NJ, U.S.A). The recording environment was a quiet room with a microphone set up to a standard distance from the mouth of $15 \mathrm{~cm}$. All participants were instructed to practise saying the target vowel at a pitch and volume with which they were comfortable three times before actual recording began. This was to optimise the degree of steadiness in phonation. The /a/ vowel was then phonated in the same way as during practice for 5 seconds. The recording was stored in digital format.

\section{Statistics}

The Statistical Package for the Social Sciences version 13.0 application for Windows (SPSS Inc., Chicago, Illinois, USA) was utilised to perform statistical analysis. For variables of a quantitative kind, mean, median and standard deviation $(\mathrm{SD})$ were recorded to capture the central tendency and dispersion of the data. The Kolmogorov-Smirnov tests allowed assessment of the degree of conformity to a normal distribution. Data for the variables obtained from the groups were compared using Student's independent t-test. The variability within groups was analysed by means of the repeated ANOVA test. The methods of evaluation used in each group were checked for correlation using Pearson's method. The level of statistical significance was set as a $\mathrm{p}$ value $<0.05$.

\section{Results}

Thirty-seven paediatric cases of bronchiectasis (14 males, 23 females) with an age range of 7 to 13 years (mean age: 11.53 years) alongside 36 healthy controls (12 boys, 24 girls), with an age range of 7 to 13 years (mean age: 9.88 years), participated in the study.

The control and case groups did not differ from each other in age or sex at the level of statistical significance.

No differences at the level of statistical significance were observable for absolute jitter, percentage jigger, shimmer or SPI. However, NHR and VTI did differ significantly between groups ( $\mathrm{p}=0.02$ and $\mathrm{p}=0.03$, respectively). Specifically, the values obtained for NHR and VTI were raised in the cases group compared to the control group.

\section{Discussion}

The principal presentation of NCFB is of obstructed airflow at a level ranging from mild to moderate and deteriorating over time. The aetiology is environmental, albeit genetics frequently determine initial susceptibility. The result is abnormal clearance of mucus by the cilia, leading to bacterial colonisation of the bronchi, setting off a chronic inflammatory response, which produces architectural changes and further failure of mucociliary function. This vicious circle then leads to more infection, greater inflammation and progressive lung destruction. The key conditions needed for bronchiectasis to occur are persistent infection and a degree of impairment in immunity.

NCFB can be confined to one area (localised) or generalised, the latter being more usual and especially in the pulmonary lobes lying inferiorly [6]. Why this should be 
so is sometimes attributed to gravitational forces acting on retained secretions. Mucociliary function is abnormal in NCFB, and infection of the sinuses and lungs therefore tends to occur. The repeated episodes of sinusitis lead to postnasal drip and production of a pus-filled sputum. Such individuals often feel the need to expectorate the sputum [7], and this action is anticipated to cause vocal alteration.

Individuals suffering from bronchiectasis may report chronic coughing (CC), that may lead to dysphonia. Actions that damage the voice, such as coughing, may produce phonotraumatic lesions, with resulting biomechanical alterations in the vocal cords, such that vocal vibration is less regular and voice signals more erratic, resulting in a more severe dysphonia [8]. The vocal alterations found with rhinosinusitis are the probable result of laryngitis provoked by persistent inflammation of the airways and repetitive trauma to the vocal cords from expectoration.

CC may present in various ways, such as voice change or other respiratory-related symptoms [9]. CC is associated with greater frequency of laryngeal lesions, abnormal laryngeal function and over-sensitive upper airways outside the chest, compared to normal [10].

Acoustic analysis is non-invasive and allows vocal function to be classified as normal or pathological [11]. Fundamental frequency $\left(\mathrm{F}_{\text {zero }}\right)$ indicates the speed at which the vocal folds are vibrating as well as the resonatory qualities of the tract above the larynx, which are influenced by where the tongue is lying and how it moves [12]. It can thus be inferred that bronchiectasis only indirectly alters the vocal tract anatomy and physiology.

Jitter and shimmer values are useful to illuminate short duration instability in the character of the voice. Whilst jitter reveals that $\mathrm{F}_{\text {zero }}$ is unstable between cycles, shimmer reveals similar instability in intensity. Jitter and shimmer reflect how hoarse and rough the voice sounds [11]. Jitter can arise due to excessive size of the vocal folds or stress thereon, from asymmetrical anatomy or abnormal muscular or neuronal function [13]. Percentage jitter is the mean variability in vocal frequency between cycles, i.e. it measures the level of perturbation in pitch. Shimmer, by contrast, measures mean difference in amplitude across a period of time, i.e. it measures the level of perturbation in amplitude. Shimmer is measured in decibels.

NHR is a measure of aperiodic noise within the recorded signal. VTI is the ratio of spectral inharmonic to harmonic energy [12].

Micro-trauma to the voice, neurological disorders and obstructive airway disease may each result in alterations to the intensity of the voice [14]. The intensity of the voice is a function of air pressure below the glottis and the air resistance of the glottis [15]. The fact that intensity is increased in cases of bronchiectasis may be explained through the pathophysiological effects of insufficient pulmonary function and incomplete obstruction of the distal airways. Moreover, CC (a very common finding in bronchiectasis) tends to promote over-adduction of the vocal folds, which may be an extra factor leading to raised VTI.

Varying amplitude and frequency in the vocal signal plus increased turbulence in the upper airway produce a raised NHR. The voice signal suffers distortion, with subharmonics starting to predominate and breaks occurring, which means that harmonics are of less importance in the signal produced [16]. The bronchiectasis cases all had raised NHR values, interpretable as vocal hoarseness and greatly adding to the impression of abnormal vocal production [14]. Dysphonia secondary to bronchiectasis may result from the following pathological features: abnormal motion of the vocal cords, postnasal drip, persistently irritated airways, pus-filled sputum, expectoration and the general impact of pooled mucus and CC.

NCFB cases, like those with disorders of the adenoids and tonsils, are prone to recurrent infective sinusitis. Mora et al. [17] undertook pre- and post-surgical acoustic evaluations of 40 paediatric cases with palatine tonsillar or adenoidal hypertrophy. The parameters measured were $\mathrm{F}_{\text {zero }}$ , jitter, shimmer, NHR, VTI, SPI, degree of voicelessness (DUV) and degree of voice breaks (DVB). The researchers observed that each one of these variables was closer to normal following adenotonsillectomy. By contrast, the present study observed differences (increases) in only two parameters: NHR and VTI.

Hamdam et al. [17] analysed vocal alteration in the short term post endotracheal intubation. The parameters examined were $\mathrm{F}_{\text {zero }}$, shimmer, NHR and VTI, before and after the procedure. There was no significant alteration in these variables, with the exception of the maximal phonation duration. In the present study, NHR and VTI being raised is attributable to $\mathrm{CC}$ and sputum production occasioning traumatic injury to the voice.

\section{Conclusion}

It has been shown that cases of bronchiectasis undergo vocal alteration at a significant level, most markedly in terms of NHR and VTI. For both these parameters, the cases 
group showed a rise in reference to the healthy controls. The study proves that bronchiectasis has an objectively demonstrable effect on vocal production and measures there-

\section{References}

1- King P1, Holdsworth S, Freezer N, Holmes P. Bronchiectasis. Intern Med J. 2006;36:729-37.

2- Goeminne P, Dupont L. Non-cystic fibrosis bronchiectasis: diagnosis and management in 21st century. Postgrad Med J 2010;86:493-501.

3- Chang AB, Redding GJ, Everard ML. State of the Art: Chronic wet cough: protracted bronchitis, chronic suppurative lung disease and bronchiectasis. Pediatric Pulmonology 2008;43:519-31.

4- Chang AB, D Bilton. Non-cystic fibrosis bronchiectasis exacerbations. Thorax 2008;63:269-76.

5- Gursel G. Does coexistence with bronchiectasis influence intensive care unit outcome in patients with chronic obstructive pulmonary disease? Heart \& Lung 2006;35: 58-65.

6- King PT, Holdsworth SR, Freezer NJ, Villanueva E, Holmes PW. Characterisation of the onset and presenting clinical features of adult bronchiectasis. Respir Med 2006;100:2183-2189

7- Nicotra MB, Rivera M, Dale AM, Shepherd R, Carter R. Clinical, pathophysiologic, and microbiologic characterization of bronchiectasis in an aging cohort. Chest 1995;108:955-961

8- Hsiao T, Liu C, Hsu C, Lin K. Vocal fold abnormalities in laryngeal tension-fatigue syndrome. J Formosan Med Assoc. 2001;100:837-40.

9- Vertigan A, Theodoros D, Gibson P, Winkworth A. Voice and upper airway symptoms in people with chronic cough and paradoxical vocal fold movement. J Voice. 2007;21:361-83. of. This fact is of importance in understanding bronchiectatic pathophysiology and associated morbidity.

10- Rolla G, Colagrande P, Magnano M, Debermardi V, Dutto L, Delpiano L, et al. Extrathoracic airway dysfunction in cough associated with gastroesophageal reflux. J Allergy Clin Immunol. 1998;102:1-11.

11- Niedzelska G. Acoustic analysis in the diagnosis of voice disorders in children. Inter J pediatric otorhinolaryngology 2001;60:119-122.

12- Mora R, Crippa B, Dellepiane M, Jankowska B. Effects of adenotonsillectomy on speech spectrum in children. Int J Pediatr Otorhinolaryngol. 2007;71:1299-1304.

13- Smith J, Rey G, Dickens P, Fletcher N, Hollemberg L, Wolfe J. Vocal tract resonances and the sound of the Australian didjeridu (yidaki). III. Determinants of playing quality. J Acoust Soc Am. 2007;121:547-58.

14- Bhuta T, Patrick L, Garnett JD. Perceptual evaluation of voice quality and its correlation with acoustic measurements. J Voice. 2004;18:299304.

15- Pontes PAL, Vieira VP, Goncalves MIR, Pontes AAL. Characteristics of hoarse, rough and normal voices: acoustic spectrographic comparative analysis. Rev Bras Otorrinolaringol 2002; 68: 182-8.

16- Hodge FS, Colton RH, Kelley RT. Vocal intensity characteristics in normal and elderly speakers. J Voice 2001;15: 503-511.

17- Hamdam AL, Sibai A, Rameh C, Kanazeh G. Short-Term Effects of Endotracheal Intubation on Voice. J Voice 2007;21:762-8.

This is an open access article distributed under the terms of the Creative Commons Attribution-NonCommercial-NoDerivs 3.0 Unported (CC BY- NC-ND3.0) Licence (http://creativecommons.org/licenses/by-nc-nd/3.0/) which permits unrestricted noncommercial use, distribution, and reproduc- tion in any medium, provided the original work is properly cited.

Please cite this article as: Calim O.F., Yıldırım Y.S., Gedik O., Ozturan O., Cakır E., Parameters Used For Objective Acoustic Vocal Analysis In Paediatric Patients with Bronchiectasis. ENT Updates 2019;9(1): 1-4. 